\title{
Tratamento de Lesão de Tronco da Artéria Coronária Esquerda após Radioterapia do Tórax
}

\author{
Treatment of Left Main Coronary Artery Lesion after Late Thoracic Radiotherapy \\ Vera Maria Cury Salemi, André L. Dabarian, Luciano Nastari, Marcus Gama, José Soares Júnior, Charles Mady \\ Instituto do Coração (InCor) do Hospital das Clínicas da Faculdade de Medicina da Universidade de São Paulo, São Paulo, Brasil
}

A prevenção de complicações cardiovasculares tardias após radioterapia (RT) para tratamento de um tumor maligno é um desafio. Relatamos o caso de um jovem paciente com linfoma de Hodgkin submetido a tratamento com RT que desenvolveu doença cardíaca isquêmica no seguimento, embora não apresentasse fatores de risco cardiovasculares. Concluímos que pacientes submetidos a RT que apresentam dor torácica deveriam ser criteriosamente avaliados em relação à doença arterial coronariana.
Prevention of late cardiovascular complications after radiation therapy (RT) for treatment of a malignant tumor is challenging. We report the case of a young male patient with Hodgkin's lymphoma treated with RT, who developed ischemic heart disease during follow-up, although he had no cardiovascular risk factors. We conclude that patients undergoing RT who experience chest pain should be fully investigated for coronary artery disease.

\section{Introdução}

A doença arterial coronariana (DAC) é uma complicação tardia após irradiação mediastinal. A doença tende a afetar o óstio coronário, presumivelmente devido à sua localização relativamente central dentro do campo de irradiação. A radioterapia (RT) pode induzir a aterosclerose acelerada, com fibrose adventícia, tecido fibrótico epicárdico e pouco material lipídico na lesão intimal, que é diferente da lesão aterosclerótica típica. Apresentamos o caso de um paciente de 23 anos com linfoma de Hodgkin (LH) que desenvolveu lesão de tronco da artéria coronária esquerda após RT terapêutica.

\section{Relato de Caso}

Paciente com 23 anos, com dor torácica atípica por 2 meses, foi admitido em nossa instituição. Ele tinha sido submetido a tratamento para linfoma de Hodgkin através de RT mediastinal e quimioterapia aos 5 anos de idade, após o qual completa remissão foi obtida. Na avaliação, o teste de esforço foi positivo, e a cintilografia com MIBI-dipiridamol mostrou defeito transitório nas paredes lateral e anterior, com fração de ejeção do ventrículo esquerdo (FEVE) normal (Figura 1). Subsequentemente, o

\section{Palavras-chave}

Vasos coronários/radioterapia, anomalias dos vasos coronários, tórax/radioterapia.

Correspondência: Vera Maria Cury Salemi •

Avenida Jandira, 185 / 41 B - Indianópolis - 04080-000 - São Paulo, SP - Brasil E-mail: verasalemi@cardiol.br, verasalemi@uol.com.br Artigo recebido em 22/12/09; revisado recebido em 14/05/10; aceito em 01/07/10. paciente foi submetido à arteriografia coronária, que revelou lesão de $80 \%$ do tronco da artéria coronária esquerda (Figura 2A). Depois disso, o paciente foi submetido à cirurgia de revascularização do miocárdio (CRM) da artéria torácica interna esquerda (ATIE) para a artéria coronária descendente anterior esquerda (DAE) e da artéria torácica interna direita (ATID) para a artéria coronária marginal esquerda. Três meses após a cirurgia, o paciente foi hospitalizado com dor torácica atípica e uma nova arteriografia mostrou oclusão da ATID. O paciente foi então submetido à intervenção coronária percutânea (ICP) com implantação de stent Infinnium (stent coronário eluído com paclitaxel) no tronco da artéria coronária esquerda (Figura 2B) com bom desfecho após 1 ano de seguimento.

\section{Discussão}

O tratamento do LH é RT combinado com quimioterapia. Nos anos 50, RT de alta voltagem era utilizada, que era mais eficaz, mas resultava em maior dano colateral. O envolvimento do coração está relacionado à dosagem e quantidade da RT, RT no lado esquerdo do tórax, bem como o local e tamanho do tumor. Alguns autores relatam que o risco cardíaco aumenta quando a dose de RT é maior do que 30 Gy e quando o coração está envolvido no campo radioativo ${ }^{1,2}$. Em vários estudos de longo prazo de pacientes submetidos a RT, as mortes resultantes de malignidades secundárias e doença cardíaca excederam aquelas causadas pelo $\mathrm{LH}^{3}$. A RT afeta o coração como um todo, levando à pericardite constritiva e também afeta o aparelho valvar, o miocárdio, o sistema de condução e a artéria coronária ${ }^{4,5}$. A incidência de DAC varia de $5,5 \%$ a $12 \%$; é a mais letal das complicações, mas é tratável ${ }^{6,7}$. O desenvolvimento da DAC após a RT demora de 3 a 29 anos (média de 13 a 16 anos) $)^{7,8}$. A RT pode induzir aterosclerose acelerada e estudos pós-morte revelaram fibrose adventícia grave, tecido fibrótico epicárdico e pouco material lipídico na lesão intimal, que é diferente da lesão aterosclerótica típica. 
Um estudo anterior mostrou aumento de 45 vezes o risco de mortalidade por infarto agudo do miocárdio em pacientes tratados com mais de 30 Gy de irradiação mediastinal antes dos 20 anos de idade ${ }^{9}$. Nosso jovem paciente tinha DAC sem apresentar fatores de risco e com histórico familiar negativo para DAC. Isso pode ser atribuído ao seu tratamento com RT 17 anos antes. A prevalência da doença cardíaca induzida por radiação (DCIR) pode variar de 6 a 30\% em pacientes

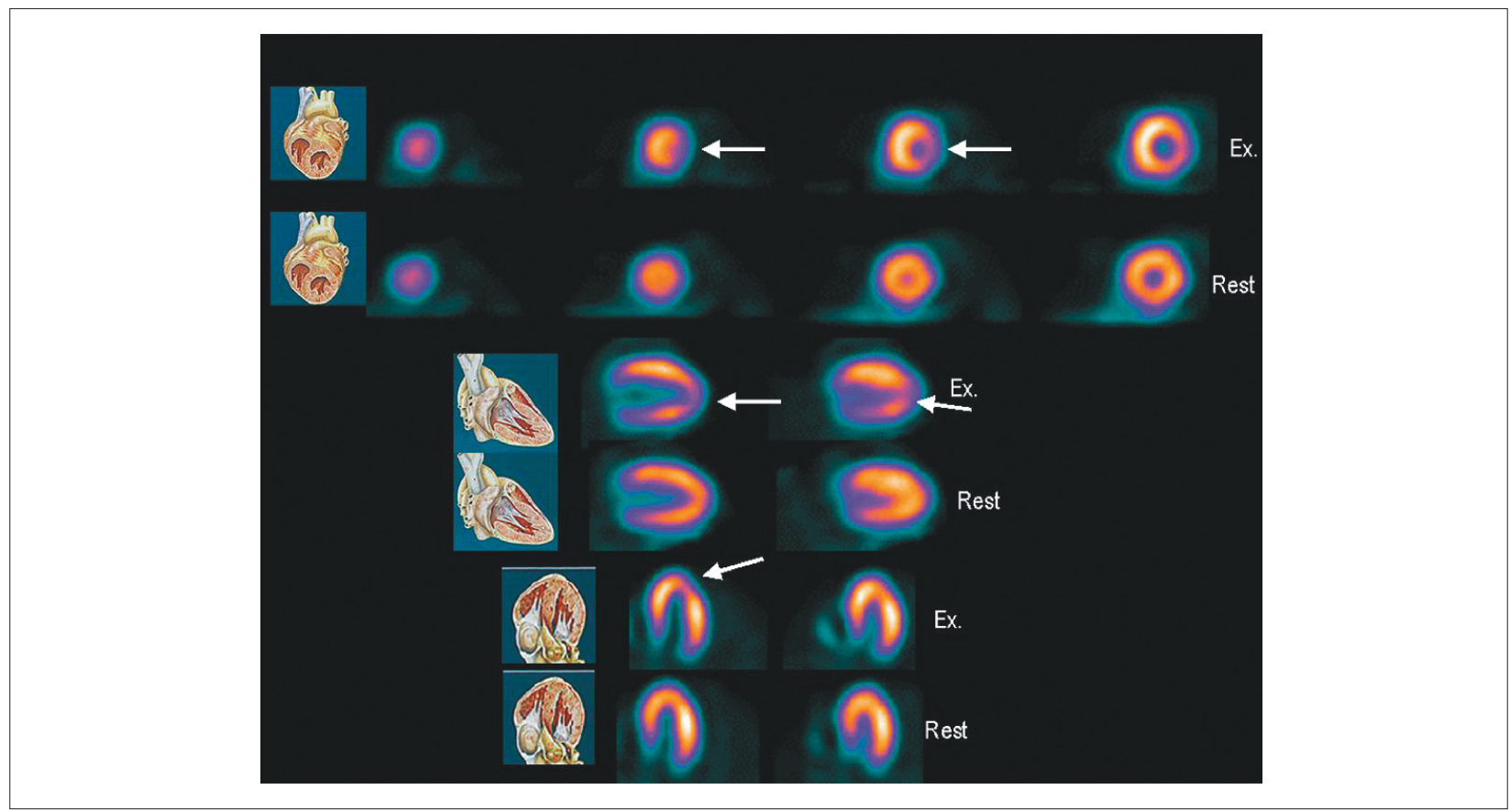

Fig. 1 - Cintilografia com MIBI-dipiridamol mostrando defeito transitório nas paredes anterior e lateral.. Ex - exercício; REP - repouso.
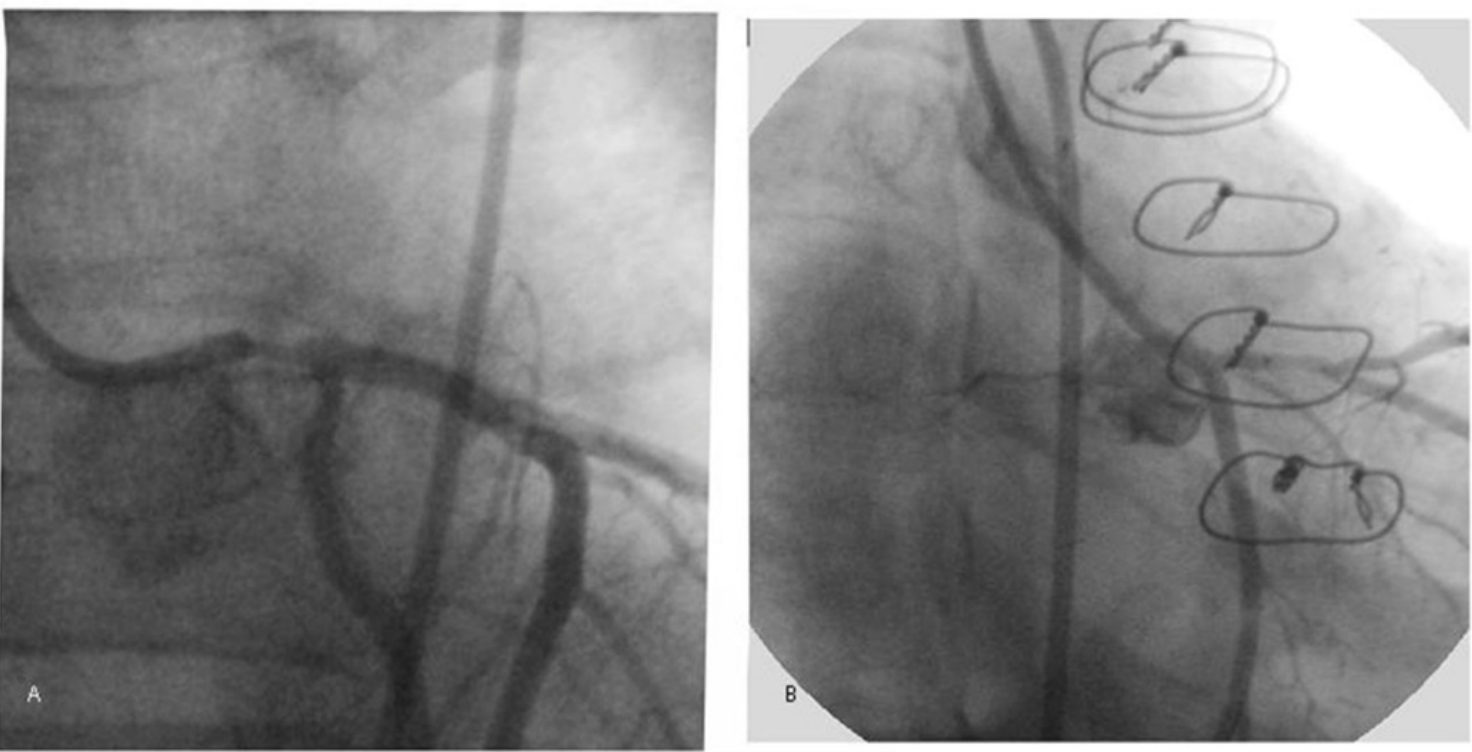

Fig. 2 - Lesão significativa do tronco da artéria coronária esquerda observada na incidência oblíqua anterior direita (A); implante de stent Infinnitum no tronco da artéria coronária esquerda com sucesso (B). 


\section{Relato de Caso}

com doença de Hodgkin. Pacientes podem apresentar fibrose miocárdica, que pode existir mesmo em indíviduos assintomáticos que receberam altas doses de RT. Um estudo de necrópsia mostrou que essa taxa é de aproximadamente $63 \%{ }^{2}$. Além disso, o envolvimento cardíaco sintomático após a RT ocorre em aproximadamente $10 \%$ dos pacientes. Está indicada a prevenção de eventos cardiovasculares, avaliação para detectar doença arterial coronariana precoce e também avaliação laboratorial completa, com especial atenção aos níveis lipídicos e função tireoidiana. A CRM é uma opção, mas a abordagem se torna difícil devido à fibrose mediastinal, que é um efeito colateral da RT anterior do tórax. Outra opção é a ICP com implante de stent, mas também é difícil, já que as lesões coronárias estão frequentemente no tronco da artéria coronária esquerda ou nas regiões proximais, levando à um maior risco para esses procedimentos ${ }^{6}$. A prevenção do dano por RT inclui a diminuição da dose total, que reduz as complicações cardíacas, mas não as malignidades secundárias. A prevenção também inclui o planejamento do tratamento através de tomografia computadorizada e a aplicação de dois campos. Além disso, a associação de RT e quimioterapia, conhecida como terapia combinada, com doses de RT de 20 a 30Gy, e, obviamente, o tratamento dos fatores de risco cardiovasculares também podem ser utilizados para tratar o dano causado pela RT. O uso de estatinas para reduzir a

\section{Referências}

1. Hull MC, Price-Mendenhall N, Colgan ME. Subdiaphragmatic Hodgkin's disease: the University of Florida experience. Int J Radiat Oncol Biol Phys. 2002;52(1):161-6.

2. Brennan S, Hann LE, Yahalom J, Oeffinger KC, Rademaker J. Imaging of late complications from mantle field radiation in lymphoma patients. Radiol Clin North Am. 2008;46(2):419-30.

3. Wethal T, Lund MB, Edvardsen T, Fosså SD, Pripp AH, Holte $\mathrm{H}$, et al. Valvular dysfunction and left ventricular changes in Hodgkin's lymphoma survivors: a longitudinal study. Br J Cancer. 2009;101(4):575-81.

4. Ng AK, Mauch PM. Late effects of Hodgkin's disease and its treatment. Cancer J. 2009;15(2):164-8.

5. Yeh ET, Bickford CL. Cardiovascular complications of cancer therapy: incidence, pathogenesis, diagnosis, and management. J Am Coll Cardiol. 2009;53(24):2231-47. inflamação e inibidores de enzima conversora de angiotensina (IECA) podem ser úteis para tratar DCIR. Adicionalmente, a pentoxifilina e alfa-tocoferol mostraram efeitos benéficos sobre a DCIR, quando iniciados antes ou três meses após a RT em ratos ${ }^{10}$.

Em conclusão, a cardiotoxicidade é uma das complicações mais importantes da terapia do câncer. À medida que mais indivíduos sobrevivem ao câncer, o trabalho da equipe composta por oncologistas e cardiologistas é importante para prevenir problemas cardíacos relacionados ao tratamento dessa doença.

\section{Potencial Conflito de Interesses}

Declaro não haver conflito de interesses pertinentes.

\section{Fontes de Financiamento}

O presente estudo não teve fontes de financiamento externas.

\section{Vinculação Acadêmica}

Não há vinculação deste estudo a programas de pósgraduação.
6. Piovaccari G, Ferretti RM, Prati F, Traini AM, Gobbi M, Caravita L, et al. Cardiac disease after chest irradiation for Hodgkin's disease: incidence in 108 patients with long follow-up. Int J Cardiol. 1995;49(1):39-43.

7. King V, Constine LS, Clark D, Schwartz RG, Muhs AG, Henzler M, et al. Symptomatic coronary artery disease after mantle irradiation for Hodgkin's disease. Int J Radiat Oncol Biol Phys. 1996;36(4):881-9.

8. Reber D, Birnbaum DE, Tollenare P. Heart diseases following mediastinal irradiation: surgical management. Eur J Cardiothorac Surg. 1995;9(4):202-5.

9. Hancock SL, Donaldson SS, Hoppe RT. Cardiac disease following treatment of Hodgkin's disease in children and adolescents. J Clin Oncol. 1993;11(7):1208-15.

10. Boerma M, Roberto KA, Hauer-Jensen M. Prevention and treatment of functional and structural radiation injury in the rat heart by pentoxifylline and alpha-tocopherol. Int J Radiat Oncol Biol Phys. 2008;72(1):170-7 